\title{
Curvature-dependent Organic Ligand Binding on Gold Nanostars Revealed by Quantitative EELS Spectral Imaging
}

Sang hyun Bae ${ }^{1}$, Pinshane Huang ${ }^{1}$, Kwahun Lee ${ }^{2}$ and Teri Odom ${ }^{2}$

${ }^{1}$ University of Illinois at Urbana-Champaign, United States, ${ }^{2}$ Northwestern University, United States

Developing quantitative nanoscale characterization methods for understanding the structure and chemistry of organic-inorganic nanoparticle interfaces is critical across wide-ranging fields including catalysis, therapeutics, and nanoelectronics. For example, gold nanostars are highly effective for the delivery of biomolecules [1,2], a property that is thought to arise from interactions between the complex curvature of the inorganic nanostars and the arrangement of molecules on the nanoparticle surface. Yet, bulk characterization techniques such as optical spectroscopy, nuclear magnetic resonance (NMR) or small angle X-ray diffraction (SAXRD) lack the spatial resolution to study how organic molecules bind to nanoparticles with complex, highly curved geometries[3]. Here, we use a combination of quantitative aberration corrected scanning transmission electron microscopy (STEM) and electron energy loss spectroscopy (EELS) spectral mapping to visualize and quantify the binding of DNA on gold nanostars (AuNS). We specifically investigated the binding of unmethylated cytosine-phosphate-guanine (CpG)coated gold nanostars, a promising system for cancer therapy[2]. Because Au nanostars contain regions of positive, negative, and neutral surface curvature, they are ideal systems to study how the geometry of the particle surfaces impacts the local binding density of organic ligand molecules on the nanoscale.

We addressed two key electron microscopy characterization challenges in this work: detecting the weak scattering signals from the light elements in DNA and developing methods to locally quantify the DNA binding density on the surface of a complex, 3D structure. The particle is supported by monolayer graphene, which we use to maximize contrast for low-Z elements[4], increase the dose resistance of the molecules,[5] and to serve as a standard for quantitative EELS[6]. To measure the DNA binding density, we used EELS spectral imaging to quantify the carbon K-edge signal at the surface of AuNS. Figure 1 shows an (a) annular-dark field(ADF) STEM image of CpG-coated AuNS and (b) accompanying EELS carbon-K edge map, in which a shell of carbon is visible around the gold particle. Next, we converted the 2D carbon maps into a local binding density on the surface of the particle by utilizing simultaneously acquired ADF and EELS data. For these measurements, we calculated the number of DNA molecules in projection using quantitative EELS measurements, then used the ADF image intensity to develop a geometric model of the particle. We validated our EELS measurements against bulk inductively coupled mass spectrometry measurements of the average per-particle $\mathrm{CpG}$ loading. Through this approach, we measured the binding density as a function of curvature across 6 different $\mathrm{CpG}$ conjugated AuNS(Figure 2). We find that, while there are large variations in binding density within and between individual particles, higher binding densities are associated with higher convex curvature. For the smallest radius of curvature we measured $(6.6 \mathrm{~nm})$, we observed that the mean $\mathrm{CpG}$ binding density is 2.2 times higher than on flat surfaces of the nanostars. These results illustrate the role of steric interactions in controlling molecular binding at soft-hard interfaces, particularly in the regime where the local radius of curvature is on the same order as the persistence length of the molecule. 


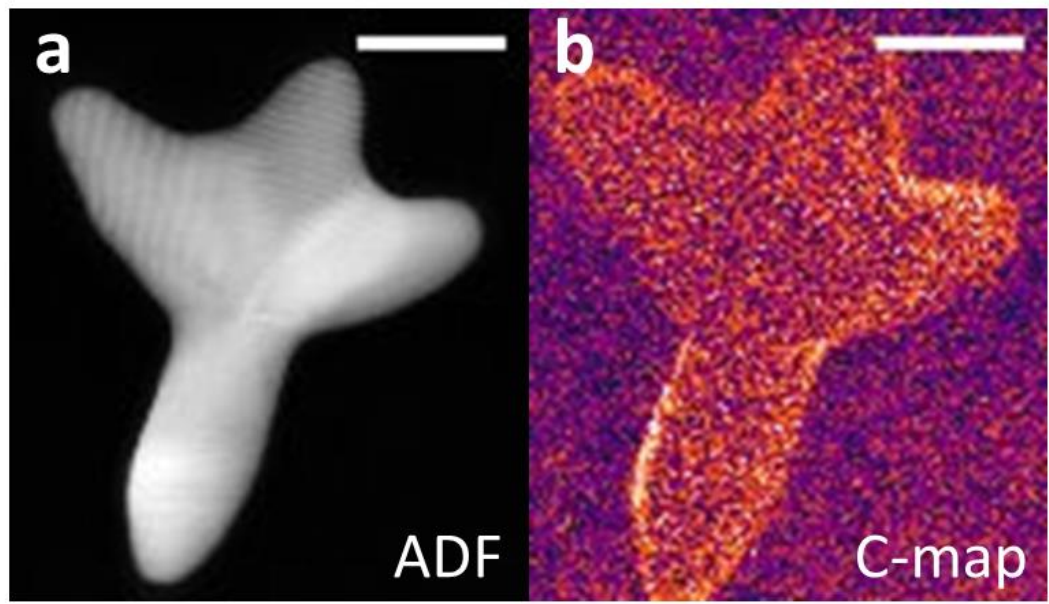

Figure 1. (a) ADF-STEM image of a single CpG conjugated gold nanostar deposited on a graphene support. (b) EELS spectral map of carbon from the same region. A carbon shell corresponding to the DNA is visible at the surface of the particle. Scale bars are $20 \mathrm{~nm}$.

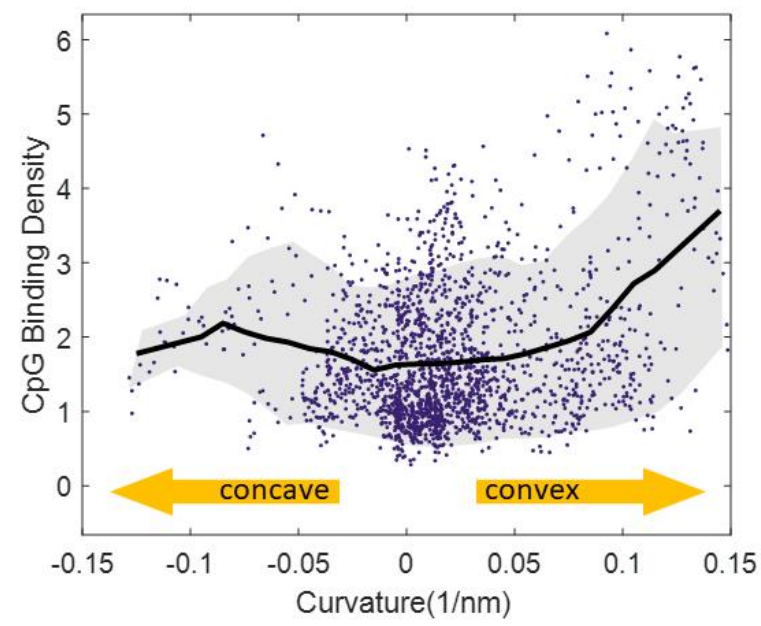

Figure 2. CpG binding density versus curvature, measured from 6 gold nanostars. The mean of the binding density at each position is plotted in black(after Savitzky-Golay smoothing), while the grey shaded region 
indicates the range that contains $90 \%$ of the plotted data. The mean binding density increases for high convex curvatures when compared to flat regions of the particles; sufficient data is not yet available to draw conclusions for the concave curvatures.

\section{References}

[1] M. Daniel, et al., Chemical Reviews 104 (2004), p. 293.

[2] K. lee, et al., Nano letters 20 (2020), p. 6170.

[3] A. Smith, et al., Analyst 142 (2017), p. 11.

[4] Z. Lee, et al., Nano letters 9 (2009), p. 3365.

[5] G. Algara-Silleret al., Applied Physics Letters 103 (2013), p. 203107.

[6] B. Janicek, et al., Nano Letters 19 (2019) p. 6308.

[7] This work was supported by NSF Division of Materials Research, grant number 1846206. The experimental work was carried out in the Materials Research Laboratory, University of Illinois Urbana-Champaign. 